\title{
Determination of Surface Currents on Circular Microstrip Antennas
}

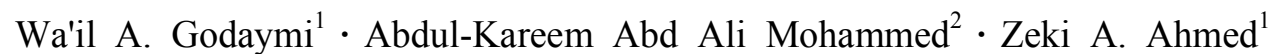

\begin{abstract}
This work aims to present a theoretical analysis of the electric and magnetic surface current densities of a circular microstrip antenna (CMSA) as a body of revolution.

The rigorous analysis of these problems begins with the application of the equivalence principle, which introduces an unknown electric current density on the conducting surface and both unknown equivalent electric and magnetic surface current densities on the dielectric surface. These current densities satisfy the integral equations (IEs) obtained by canceling the tangential components of the electric field on the conducting surface and enforcing the continuity of the tangential components of the fields across the dielectric surface. The formulation of the radiation problems is based on the combined field integral equation. This formulation is coupled with the method of moments (MoMs) as a numerical solution for this equation.

The numerical results of the electric and magnetic surface current densities on the outside boundary of a CMSA excited by $T M_{11}-$ and $T M_{21}-$ modes are presented. The radiation pattern is calculated numerically in the two principle planes for a CMSA and gives a good results compared with measured results published by other research workers.

Key words: Microstrip Antennas, Body of Revolutions.
\end{abstract}

\section{Introduction}

A microstrip antenna (MSA) consists of a very thin metallic strip (patch) placed on a small fraction of a wavelength above a ground plane and separated from the ground plane by a dielectric sheet. The radiating elements and the feed line are usually photoetched on the dielectric substrate. The radiating patch may be square, rectangular, circular, or any other geometry. These patch shapes are the most common because of their ease of analysis and fabrication. The feed line is often a conducting strip, usually of a smaller width; however, coaxial line feeds, where the inner conductor is attached to the radiating patch, are also widely used [1].

The radiation of patch antennas is efficient only when the excitation is made at the resonant frequency of a mode. The field is usually dominated by that single mode in the frequency range of interest [2].

For a mobile communications system, the technique of printed antennas seems to have numerous advantages. Therefore, printed antennas have gained more and more importance in the field of antenna design. This led us to the decision to implement a microstrip antenna [3].

Most analyses of MSA characteristics assume a flat, perfectly conducting ground plane of infinity area. However, in practice, the ground plane may not be flat or perfectly conducting; it certainly does not have an infinite extent. The size and shape of a small ground plane can have significant effects on the electrical characteristics of the antenna, such as radiation pattern.

Among the various shapes of MSAs, the circular patches are the ones that have been more extensively studied. However, in some applications such as arrays, circular geometries offer certain advantages over other configurations. Recent experiment results have shown that circular disk microstrip elements may be easily modified to produce a range of impedances, radiation patterns, and frequencies of operation [1].

The general formulation starts with the application of the concept of equivalence principle and the provision of IEs for field distribution on the surfaces of the conductors and dielectric substrate. This introduces an unknown electric surface current density on the conducting surfaces and unknown equivalent electric and magnetic

Manuscript received September 24, 2012 ; Revised November 15, 2012 ; Accepted November 28, 2012. (ID No. 20120924-018J)

${ }^{1}$ Dept. of Physics, College of Science, Univ. of Basrah, Basrah, Iraq.

${ }^{2}$ Atmosphere and Space Science Center, Ministry of Science and Technology, Baghdad, Iraq.

Corresponding Author : Zeki A. Ahmed (e-mail : zeki abduallah@yahoo.com)

This is an Open-Access article distributed under the terms of the Creative Commons Attribution Non-Commercial License (http://creativecommons.org/licenses/ by-nc/3.0) which permits unrestricted non-commercial use, distribution, and reproduction in any medium, provided the original work is properly cited. 
surface current densities on the dielectric surface. These currents, with the impressed current in the dielectric region, are the sources of the electric and magnetic fields in the presented regions. The formulation is exact and satisfies all boundary conditions. The boundary conditions are applied on the various surfaces of the MSA.

The IEs are solved numerically using the MoMs [4]. Numerical results for the surface magnetic and electric current densities are presented for both finite [5] and infinite ground planes [1]. The usefulness of the method for solving the problem of a CMSA is discussed.

\section{Formulation of a CMSA Problem}

The EM problem involving MSAs deals with the determination of the field components in the presence of conductors and dielectrics. Thus, the boundary conditions to be satisfied are of mixed type. This requires the vanishing of the tangential electric field components in the conductors and the continuity of the tangential electric and magnetic components in the dielectrics. An exact analytical solution cannot be found to satisfy all boundary conditions because practical geometries are finite in size. Therefore, a numerical solution must be utilized. For homogeneous dielectrics, a convenient formulation can be developed in terms of the tangential field components on the boundary surfaces. The resulting IE includes all boundary conditions. Thus, the formulation of the problem is in terms of the surface integral equations, which are exact. Consequently, the accuracy of the solution depends on the nature of the numerical technique selected to solve them and used to determine the unknown surface distributions.

The CMSA can be considered a structure through which power is coupled to an exterior region (free space) via an aperture formed by the dielectric surface, as shown in Fig. 1, where a homogeneous dielectric material is sandwiched between two conducting layers. The sources of the EM excitation are provided by the impressed

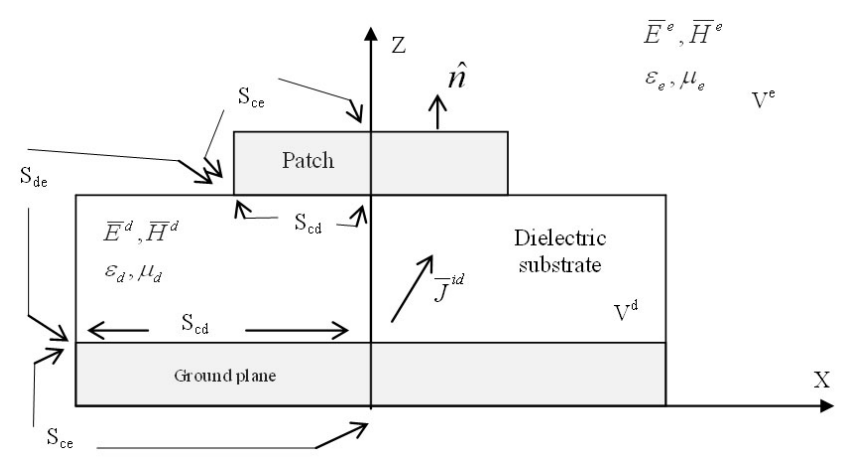

Fig. 1. Geometric of a circular microstrip antenna. electric current $\bar{J}^{i d}$ in the dielectric region.

In this analysis, two regions are defined to represent the CMSA radiation in free space. First, a finite region of space of volume $V^{d}$ is filled with a homogeneous material of permeability $\mu_{d}$ and permittivity $\varepsilon_{d}$ with an impressed electric current source of density $\bar{J}^{i d}$. Second, an infinite region of volume $V^{e}$ is filled with a homogenous material of permeability $\mu_{e}$ and permittivity $\varepsilon_{e}$. The subscripts and superscripts $d$ and $e$ refer to dielectric and empty, respectively.

The surfaces $S_{c e}$ and $S_{c d}$ are the boundaries to the conducting regions. Neither $S_{c e}$ nor $S_{c d}$ is a single surface, but they are two separated surfaces below and above the dielectric. $S_{d e}$ is the dielectric surface on which the regions $V^{d}$ and $V^{e}$ are coupled. The EM field in $V^{d}$ is denoted by $\bar{E}^{d}, \bar{H}^{d}$ and that in $V^{e}$ by $\bar{E}^{e}$ and $\bar{H}^{e}$. In the present work, all these surfaces are assumed to be rotationally symmetric to represent bodies of revolutions (BoRs).

To obtain the solution for the electric and magnetic fields in both regions mentioned above, the equivalence principle is used to acquire the auxiliary problems shown in Figs. 2 and 3. In this principle, the CMSA is divided into two equivalent regions $V^{e}$ and $V^{d}$.

The boundary conditions to be satisfied are:-

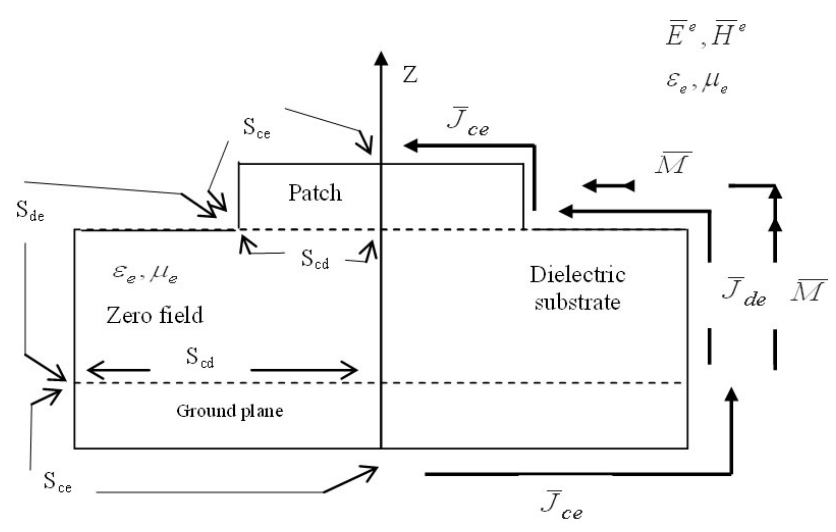

Fig. 2. Equivalent for region $V^{e}$.

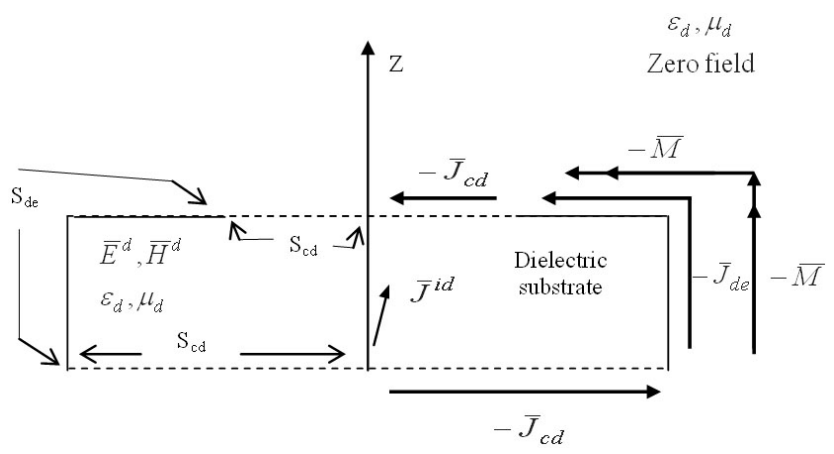

Fig. 3. Equivalent for region $V^{d}$. 


$$
\begin{array}{ll}
\hat{n} \times \bar{E}^{e}=0 & \text {, on } S_{c e} \\
\hat{n} \times \bar{E}^{e}=0 & \text {, on } S_{c d} \\
\hat{n} \times \bar{E}^{d}=\hat{n} \times \bar{E}^{e} & \text {, on } S_{d e} \\
\hat{n} \times \bar{H}^{d}=\hat{n} \times \bar{H}^{e} & \text {, on } S_{d e}
\end{array}
$$

and the surface equivalent currents are:-

$$
\begin{array}{ll}
\bar{J}_{c e}=\hat{n} \times \bar{H}^{e} & \text {, on } S_{c e} \\
\bar{J}_{c d}=\hat{n} \times \bar{H}^{d} & \text {, on } S_{c d} \\
\bar{J}_{d e}=\hat{n} \times \bar{H}^{e} & \text {, on } S_{d e} \\
\bar{M}=-\hat{n} \times \bar{E}^{e} & \text {, on } S_{d e}
\end{array}
$$

The following set of equations, which is the mathematical representation of the equivalence regions, is sufficient to determine $\bar{J}_{c e}, \bar{J}_{c d}, \bar{J}_{d e}$, and $\bar{M}$ [6]:-

$$
\begin{aligned}
& \hat{n} \times \bar{E}^{e}\left(\bar{J}_{c e}+\bar{J}_{d e}, \bar{M}\right)=07 \text { on } S_{c e} \text { and } S_{d e} \\
& \left.\hat{n} \times \bar{H}^{e}\left(\bar{J}_{c e}+\bar{J}_{d e}, \bar{M}\right)=0\right] \text { in Fig. } 2 \\
& \hat{n} \times \bar{E}^{d}\left(-\bar{J}_{c d}-\bar{J}_{d e},-\bar{M}\right)+\hat{n} \times \bar{E}^{d}\left(\bar{J}^{i d}, 0\right)=0 \\
& \hat{n} \times \bar{H}^{d}\left(-\bar{J}_{c d}-\bar{J}_{d e},-\bar{M}\right)+\hat{n} \times \bar{H}^{d}\left(\bar{J}^{i d}, 0\right)=0
\end{aligned}
$$

just outside $S_{c d}$ and $S_{d e}$ in Fig. 3

where $\bar{E}^{a}(\bar{J}, \bar{M})$ and $\bar{H}^{a}(\bar{J}, \bar{M})$ are the electric and magnetic fields due to the equivalent electric and magnetic currents $\bar{J}$ and $\bar{M}$ radiating in the homogeneous medium characterized by $\mu_{a}$ and $\varepsilon_{a}$ everywhere, respectively. The superscript (a) represents (e) or (d), whereas $\bar{E}^{d}\left(\bar{J}^{i d}, 0\right)$ and $\bar{H}^{d}\left(\bar{J}^{i d}, 0\right)$ are the excitation electric and magnetic fields, respectively, caused by the impressed electric current density $\bar{J}^{i d}$.

One set of equations that can be identified from Eqs. (3) is given by:-

$$
\left.\begin{array}{l}
\bar{E}_{\text {tan }}^{e}\left(\bar{J}_{c e}+\bar{J}_{d e}, \bar{M}\right)=0, \quad \text { on } S_{c e} \\
\bar{E}_{\text {tan }}^{d}\left(\bar{J}_{c d}+\bar{J}_{d e}, \bar{M}\right)=\bar{E}_{\text {tan }}^{d}\left(\bar{J}^{i d}\right), \text { on } S_{c d} \\
\bar{E}_{\text {tan }}^{e}\left(\bar{J}_{c e}+\bar{J}_{d e}, \bar{M}\right)+\bar{E}_{\text {tan }}^{d}\left(\bar{J}_{c d}+\bar{J}_{d e}, \bar{M}\right)=\bar{E}_{\text {tan }}^{d}\left(\bar{J}^{i d}\right) \\
\bar{H}_{\text {tan }}^{e}\left(\bar{J}_{c e}+\bar{J}_{d e}, \bar{M}\right)+\bar{H}_{\text {tan }}^{d}\left(\bar{J}_{c d}+\bar{J}_{d e}, \bar{M}\right)=\bar{H}_{\text {tan }}^{d}\left(\bar{J}^{i d}\right)
\end{array}\right]
$$

The subscript $(\tan )$ denotes the tangential components of the fields on the surface in the equation. These boundary conditions, Eq. (4), are sufficient to determine the fields $\bar{E}^{d}, \bar{E}^{e}, \bar{H}^{d}$, and $\bar{H}^{e}$.

Eqs. (4) represents the IEs for these currents. These
IEs can be generated using appropriate vector potentials in terms of which the field vectors are given by Ref. [7]:-

$$
\begin{aligned}
& \bar{E}^{a}(\bar{J}, \bar{M})=-j \omega \bar{A}^{a}(\bar{J})-\nabla \Phi^{a}(\bar{J})-\frac{1}{\varepsilon_{a}} \nabla \times \bar{F}^{a}(\bar{M}) \\
& \bar{H}^{a}(\bar{J}, \bar{M})=-j \omega \bar{F}^{a}(\bar{M})-\nabla \psi^{a}(\bar{M})+\frac{1}{\mu_{a}} \nabla \times \bar{A}^{a}(\bar{J})
\end{aligned}
$$

where $\bar{A}^{a}(\bar{J})$ and $\bar{F}^{a}(\bar{M})$ are the magnetic and electric vector potentials, respectively, given by-

$$
\begin{aligned}
& \bar{A}^{a}(\bar{J})=\mu_{a} \int_{s^{\prime}} \bar{J}\left(\bar{r}^{\prime}\right) G^{a}\left(\bar{r}, \bar{r}^{\prime}\right) d s^{\prime} \\
& \bar{F}^{a}(\bar{M})=\varepsilon_{a} \int_{s^{\prime}} \bar{M}\left(\bar{r}^{\prime}\right) G^{a}\left(\bar{r}, \bar{r}^{\prime}\right) d s^{\prime}
\end{aligned}
$$

While, $\Phi^{a}(\bar{J})$ and $\psi^{a}(\bar{M})$ are the electric and magnetic scalar potential, respectively, given by :-

$$
\begin{aligned}
& \Phi^{a}(\bar{J})=\frac{1}{\varepsilon_{a}} \int_{s^{\prime}} \sigma(\bar{J}) G^{a}\left(\bar{r}, \bar{r}^{\prime}\right) d s^{\prime} \\
& \psi^{a}(\bar{M})=\frac{1}{\mu_{a}} \int_{s^{\prime}} m(\bar{M}) G^{a}\left(\bar{r}, \bar{r}^{\prime}\right) d s^{\prime}
\end{aligned}
$$

and,

$$
\begin{aligned}
& \sigma(\bar{J})=\frac{j}{\omega} \bar{\nabla}_{s}^{\prime} \cdot \bar{J}\left(\bar{r}^{\prime}\right) \\
& m(\bar{M})=\frac{j}{\omega} \bar{\nabla}_{s}^{\prime} \cdot \bar{M}\left(\bar{r}^{\prime}\right)
\end{aligned}
$$

where, $\sigma(\bar{J})$ is the electric surface charge, $m(\bar{M})$ is the magnetic surface charge and $\omega$ is the angular frequency. The function $G^{a}\left(\bar{r}, \bar{r}^{\prime}\right)$ is the scalar Greens function and is given by :-

$$
G\left(\bar{r}, \bar{r}^{\prime}\right)=\frac{e^{-j k\left|\bar{r}-\bar{r}^{\prime}\right|}}{4 \pi\left|\bar{r}-\bar{r}^{\prime}\right|}
$$

where $k=\omega \sqrt{\mu \varepsilon}$ is the wave number and $\omega$ is the fixed angular frequency of the problem.

\section{2-1 Formulation of the Analysis}

The coordinate system of the CMSA considered in this analysis as a simple representation of an axisymmetric object is shown in Fig. (4). In this figure, the notations without prime represent the field point, whereas the notations with prime represent the source point. We define the orthogonal surface tangents by their unit vectors $\hat{u}_{t}$ and $\hat{u}_{\phi}$ and the outward normal by the direction of its unit vector $\hat{u}_{n}$ given by-

$$
\hat{u}_{n}=\hat{u}_{\phi} \times \hat{u}_{t}
$$




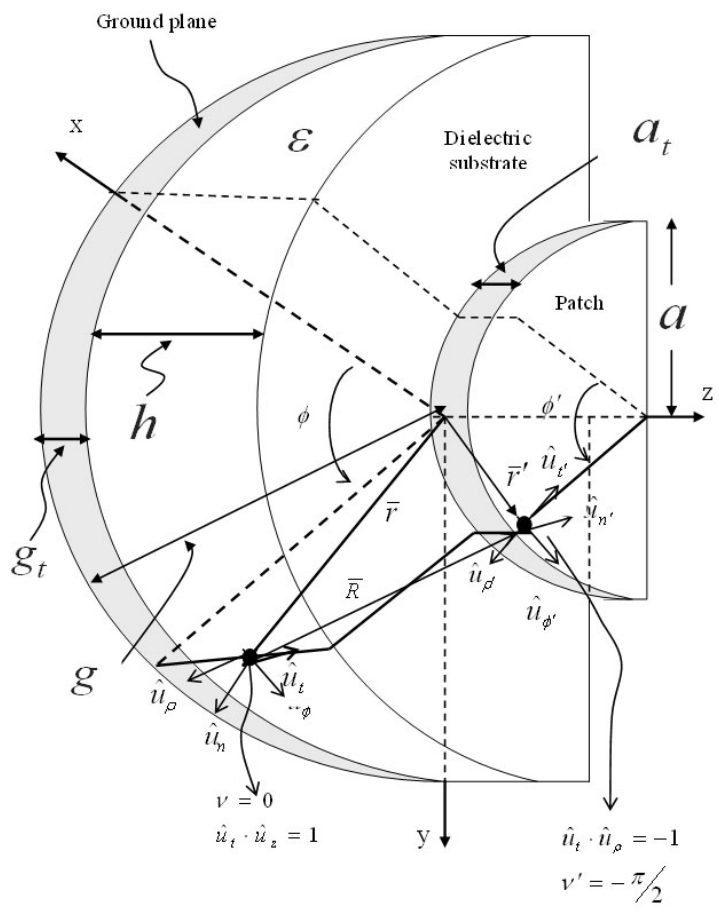

Fig. 4. Geometry of the circular microstrip as a body of revolution. patch of radius $(a)$ and thickness $\left(a_{t}\right)$, ground plane of radius $(g)$ and thickness $\left(g_{t}\right)$ and dielectric material of thickness $(h)$.

These unit vectors can be determined in cylindrical coordinates by :-

$$
\begin{aligned}
\hat{u}_{t}= & \hat{u}_{\rho} \sin v+\hat{u}_{z} \cos v \\
\hat{u}_{\phi}= & \hat{u}_{\phi} \\
\hat{u}_{t^{\prime}}= & \hat{u}_{\rho} \sin v^{\prime} \cos \left(\phi^{\prime}-\phi\right) \\
& +\hat{u}_{\phi} \sin v^{\prime} \sin \left(\phi^{\prime}-\phi\right)+\hat{u}_{z} \cos v^{\prime} \\
\hat{u}_{\phi^{\prime}}= & -\hat{u}_{\rho} \sin \left(\phi^{\prime}-\phi\right)+\hat{u}_{\phi} \cos \left(\phi^{\prime}-\phi\right)
\end{aligned}
$$

where $v$ is the angle between the $\hat{u}_{t}$ and the z-axis, which is positive if $\hat{u}_{t}$ points away from the $\mathrm{z}$-axis and negative if $\hat{u}_{t}$ points toward it. Similarly, $v^{\prime}$ is the angle between $\hat{u}_{t}^{\prime}$ and the z-axis at $\left(t^{\prime}, \phi^{\prime}\right)$.

If the positional vectors of points $(t, \phi)$ and $\left(t^{\prime}, \phi^{\prime}\right)$ are $\bar{r}$ and $\bar{r}^{\prime}$, respectively, then we can obtain the relative positional vector $\bar{R}$ [see Fig. 4] to have the following form:-

$$
|\bar{R}|=\left[\left(\rho-\rho^{\prime}\right)^{2}+\left(z-z^{\prime}\right)^{2}+4 \rho \rho^{\prime} \sin ^{2}\left(\frac{\phi^{\prime}-\phi}{2}\right)\right]^{1 / 2}
$$

\section{2-2 Method of Solution}

The surfaces of the CMSA can be generated by rotating a generating curve, which is a plane curve, about the z-axis. In the computation, the generating curve of the surfaces $\left(S_{c e}+S_{d e}\right)$ and $\left(S_{c d}+S_{d e}\right)$ is approximated by choosing a succession of points $t_{i}(i=1,2, \cdots N)$ on the generating curve and then connecting these points by straight-line segments ( $N$ represents the total number of points). The point $t_{i}$ is called a data point; its location is specified by its distance $\rho_{i}$ from the z-axis and its $z_{i}$ coordinates.

The number of data points on the patch, dielectric, and ground plane surfaces is $N_{p}, N_{d}$, and $N_{g}$, respectively. The first data point on dielectric surface $S_{d e}$ is the point where $S_{d e}$ meets the ground plane. This data point is the $\left(N_{g}+1\right)$ on the generating curve. The last data point on $S_{d e}$ is the point where $S_{d e}$ meets the conducting patch.

\section{2-2-1 Expansion of the Unknown Currents}

According to the MoM procedures, which reduce Eqs. (5) and (6) to a set of linear equations in matrix form, the unknown surface currents on the different surfaces of the CMSA are expressed in a finite modal expansion [8]. On a BoR, the induced currents have components along two orthogonal surface vectors $\hat{u}_{t}$ and $\hat{u}_{\phi}$. The electric and magnetic current densities can now be decomposed into two components along the unit vector $\hat{u}_{t^{\prime}}$ and $\hat{u}_{\phi^{\prime}}$ and expressed in the following form [9], [10]:-

$$
\begin{aligned}
& \bar{J}\left(\bar{r}^{\prime}\right)=\sum_{n=-\infty}^{\infty}\left(\bar{J}_{n}^{t}\left(t^{\prime}\right)+\bar{J}_{n}^{\phi}\left(t^{\prime}\right)\right) e^{j n \phi^{\prime}} \\
& \bar{M}\left(\bar{r}^{\prime}\right)=\eta_{e} \sum_{n=-\infty}^{\infty}\left(\bar{M}_{n}^{t}\left(t^{\prime}\right)+\bar{M}_{n}^{\phi}\left(t^{\prime}\right)\right) e^{j n \phi^{\prime}}
\end{aligned}
$$

where $\eta_{e}$ is the free space impedance.

The electric current $\bar{J}$ exists on both conducting and dielectric surfaces, where $\bar{M}$ exists only on the dielectric. The variation $\phi^{\prime}$ is known as a continuous sinusoidal distribution with $e^{j n \phi^{\prime}}$, and the $t^{\prime}$ variation is an unknown function that can be expanded as a linear combination of $N$ terms as:-

$$
\begin{aligned}
& \bar{J}_{n}^{\alpha}\left(t^{\prime}\right)=\hat{u}_{\alpha^{\prime}} \sum_{i=1}^{N-6} I_{n i}^{\alpha} f_{i}\left(t^{\prime}\right) \\
& \bar{M}_{n}^{\alpha}\left(t^{\prime}\right)=\hat{u}_{\alpha^{\prime}} \sum_{i=1}^{N_{d}-2} K_{n i}^{\alpha} f_{i}\left(t^{\prime}\right)
\end{aligned}
$$

where $\alpha$ is the $t$ or $\phi$-component.

The range of the integer $\mathrm{n}$ gives the total number of azimuthal modes. Here, $I_{n i}^{t}, I_{n i}^{\phi}, K_{n i}^{t}$, and $K_{n i}^{\phi}$ are the unknown current coefficients to be found, and $f_{i}\left(t^{\prime}\right)$ is the triangle function. Through a simple derivation, one can 
yield the following:-

$$
\begin{gathered}
\sum_{n=-\infty}^{\infty}\left[\sum_{i=1}^{2\left(N-N_{d}-4\right)} I_{n i}^{1 e} \bar{E}_{\text {tan }}^{e}\left(\bar{J}_{n i}^{1 e}, 0\right)+\sum_{i=1}^{2\left(N_{d}-2\right)} I_{n i} \bar{E}_{\tan }^{e}\left(\bar{J}_{n i}^{2 e}, 0\right)\right. \\
\left.+\eta_{e} \sum_{i=1}^{2\left(N_{d}-2\right)} K_{n i} \bar{E}_{\text {tan }}^{e}\left(0, \bar{M}_{n i}\right)\right]=0
\end{gathered}
$$

, on $S_{c e}$

$$
\begin{aligned}
& \sum_{n=-\infty}^{\infty}\left[\sum_{i=1}^{2\left(N-N_{d}-4\right)} I_{n i}^{1 d} \bar{E}_{\mathrm{tan}}^{d}\left(\bar{J}_{n i}^{1 d}, 0\right)+\sum_{i=1}^{2\left(N_{d}-2\right)} I_{n i} \bar{E}_{\mathrm{tan}}^{d}\left(\bar{J}_{n i}^{2 d}, 0\right)\right. \\
& \left.+\eta_{e} \sum_{i=1}^{2\left(N_{d}-2\right)} K_{n i} \bar{E}_{\mathrm{tan}}^{d}\left(0, \bar{M}_{n i}\right)\right]=\bar{E}_{\mathrm{tan}}^{d}\left(\bar{J}^{i d}\right)
\end{aligned}
$$

, on $S_{c d}$

$$
\begin{aligned}
& \sum_{n=-\infty}^{\infty}\left[\sum_{i=1}^{2\left(N-N_{d}-4\right)}\left\{I_{n i}^{1 e} \bar{E}_{\text {tan }}^{e}\left(\bar{J}_{n i}^{1 e}, 0\right)+I_{n i}^{1 d} \bar{E}_{\text {tan }}^{d}\left(\bar{J}_{n i}^{1 d}, 0\right)\right\}\right. \\
& +\sum_{i=1}^{2\left(N_{d}-2\right)}\left\{I_{n i} \bar{E}_{\text {tan }}^{e}\left(\bar{J}_{n i}^{2 e}, 0\right)+I_{n i} \bar{E}_{\text {tan }}^{d}\left(\bar{J}_{n i}^{2 d}, 0\right)\right\} \\
& \left.+\eta_{e} \sum_{i=1}^{2\left(N_{d}-2\right)}\left\{K_{n i} \bar{E}_{\text {tan }}^{e}\left(0, \bar{M}_{n i}\right)+K_{n i} \bar{E}_{\text {tan }}^{d}\left(0, \bar{M}_{n i}\right)\right\}\right]=\bar{E}_{\text {tan }}^{d}\left(\bar{J}^{i d}\right)
\end{aligned}
$$$$
\text { , on } S_{d e}
$$

$$
\begin{aligned}
& \sum_{n=-\infty}^{\infty}\left[\sum_{i=1}^{2\left(N-N_{d}-4\right)}\left\{I_{n i}^{1 e} \bar{H}_{\text {tan }}^{e}\left(\bar{J}_{n i}^{l e}, 0\right)+I_{n i}^{1 d} \bar{H}_{\text {tan }}^{d}\left(\bar{J}_{n i}^{1 d}, 0\right)\right\}\right. \\
& \quad+\sum_{i=1}^{2\left(N_{d}-2\right)}\left\{I_{n i} \bar{H}_{\text {tan }}^{e}\left(\bar{J}_{n i}^{2 e}, 0\right)+I_{n i} \bar{H}_{\text {tan }}^{d}\left(\bar{J}_{n i}^{2 d}, 0\right)\right\}+ \\
& \left.\quad+\eta_{e} \sum_{i=1}^{2\left(N_{d}-2\right)}\left\{K_{n i} \bar{H}_{\text {tan }}^{e}\left(0, \bar{M}_{n i}\right)+K_{n i} \bar{H}_{\text {tan }}^{d}\left(0, \bar{M}_{n i}\right)\right\}\right]=\bar{H}_{\text {tan }}^{d}\left(\bar{J}^{i d}\right) \\
& \quad, \text { on } S_{d e}
\end{aligned}
$$

\section{2-3 Weighting Functions}

The choice of weighting functions $W$ is important in that the elements of this functions must be linearly independent to make the resulting equations linearly independent. Furthermore, choosing weighting functions that minimize the computations required to evaluate the inner products is generally advantageous. As such, similar types of functions are often used for both weighting and expansion functions (i.e., $W=J^{*}$, where $*$ denotes a complex conjugate); this is known as the Galerkin method [4]. The testing functions are defined by-

$$
\begin{aligned}
& \bar{W}(\bar{r})=\bar{W}^{t}(t, \phi)+\bar{W}^{\phi}(t, \phi)=\sum_{m=-\infty}^{\infty} \sum_{i=1}^{N-6}\left[\bar{W}_{m i}^{t}(t, \phi)+\bar{W}_{m i}^{\phi}(t, \phi)\right] \\
& \bar{W}_{m i}^{\alpha}(t, \phi)=\hat{u}_{\alpha} f_{i}(t) e^{-j m \phi}
\end{aligned}
$$

The expansion and testing functions $\left(\left[\bar{J}_{n i}, \bar{M}_{n i}\right]\right.$ and $\left[\bar{W}_{m i}\right\rfloor$ ), as defined by Eqs. (17), (20b), and (20c), are orthogonal over the period 0 to $2 \pi$ in $\phi$, for $(n \neq m)$. This indicates that the inner products of these functions disappear for $(n \neq m)$. This fact allows each mode to be treated completely independent of the other modes. Accordingly, we obtain a separate matrix equation for each mode.

The inner product of the electric and magnetic field equations, given by Eqs. (19), is taken at each side by a set of testing function. To select the appropriate values for the elements of the testing functions in the inner product of Eqs. (19), it is possible to note down the following:-

$$
\text { 2-3-1 For } j=1,2,3, \cdots \cdots ., 2\left(\mathrm{~N}-\mathrm{N}_{d}-4\right)
$$

$$
\begin{aligned}
& \sum_{i=1}^{2\left(N-N_{d}-4\right)} I_{n i}^{1 e}\left\langle\bar{W}_{n j}^{1 e}, \bar{E}_{\text {tan }}^{e}\left(\bar{J}_{n i}^{1 e}, 0\right)\right\rangle+\sum_{i=1}^{2\left(N_{d}-2\right)} I_{n i}\left\langle\bar{W}_{n j}^{1 e}, \bar{E}_{\text {tan }}^{e}\left(\bar{J}_{n i}^{2 e}, 0\right)\right\rangle \\
& \quad+\eta_{e} \sum_{i=1}^{2\left(N_{d}-2\right)} K_{n i}\left\langle\bar{W}_{n j}^{1 e}, \bar{E}_{\text {tan }}^{e}\left(0, \bar{M}_{n i}\right)\right\rangle=0 \\
& \quad, \text { on } S_{c e} \\
& \sum_{i=1}^{2\left(N-N_{d}-4\right)} I_{n i}^{1 d}\left\langle\bar{W}_{n j}^{1 d}, \bar{E}_{\text {tan }}^{d}\left(\bar{J}_{n i}^{1 d}, 0\right)\right\rangle+\sum_{i=1}^{2\left(N_{d}-2\right)} I_{n j}\left\langle\bar{W}_{n i}^{1 d}, \bar{E}_{\text {tan }}^{d}\left(\bar{J}_{n i}^{2 d}, 0\right)\right\rangle \\
& +\eta_{e} \sum_{i=1}^{2\left(N_{d}-2\right)} K_{n i}\left\langle\bar{W}_{n j}^{1 d}, \bar{E}_{\text {tan }}^{d}\left(0, \bar{M}_{n i}\right)\right\rangle=\left\langle\bar{W}_{n j}^{1 d}, \bar{E}_{\text {tan }}^{d}\left(\bar{J}^{i d}\right)\right\rangle \\
& \text {, on } S_{c d} \quad(21 \mathrm{~b})
\end{aligned}
$$

2-3-2 For $j=1,2,3, \cdots \cdots ., 2\left(\mathrm{~N}_{d}-2\right)$

$$
\begin{aligned}
& \sum_{i=1}^{2\left(N-N_{d}-4\right)}\left[I_{n i}^{1 e}\left\langle\bar{W}_{n j}^{2 e}, \bar{E}_{\tan }^{e}\left(\bar{J}_{n i}^{1 e}, 0\right)\right\rangle+I_{n i}^{1 d}\left\langle\bar{W}_{n j}^{2 d}, \bar{E}_{\text {tan }}^{d}\left(\bar{J}_{n i}^{1 d}, 0\right)\right\rangle\right] \\
& +\sum_{i=1}^{2\left(N_{d}-2\right)} I_{n i}\left[\left\langle\bar{W}_{n j}^{2 e}, \bar{E}_{\tan }^{e}\left(\bar{J}_{n i}^{2 e}, 0\right)\right\rangle\right. \\
& \left.+\left\langle\bar{W}_{n j}^{2 d}, \bar{E}_{\text {tan }}^{d}\left(\bar{J}_{n i}^{2 d}, 0\right)\right\rangle\right]+\eta_{e} \sum_{i=1}^{2\left(N_{d}-2\right)} K_{n i}\left[\left\langle\bar{W}_{n j}^{2 e}, \bar{E}_{\text {tan }}^{e}\left(0, \bar{M}_{n i}\right)\right\rangle\right. \\
& \left.+\left\langle\bar{W}_{n j}^{2 d}, \bar{E}_{\text {tan }}^{d}\left(0, \bar{M}_{n i}\right)\right\rangle\right]=\left\langle\bar{W}_{n j}^{2 d}, \bar{E}_{\text {tan }}^{d}\left(\bar{J}^{i d}\right)\right\rangle
\end{aligned}
$$

, on $S_{d e}$

$$
\begin{aligned}
& \sum_{i=1}^{2\left(N-N_{d}-4\right)}\left[I_{n i}^{1 e}\left\langle\bar{W}_{n j}^{3}, \bar{H}_{\mathrm{tan}}^{e}\left(\bar{J}_{n i}^{1 e}, 0\right)\right\rangle+I_{n i}^{1 d}\left\langle\bar{W}_{n j}^{3}, \bar{H}_{\mathrm{tan}}^{d}\left(\bar{J}_{n i}^{1 d}, 0\right)\right\rangle\right] \\
& +\sum_{i=1}^{2\left(N_{d}-2\right)} I_{n i}\left[\left\langle\bar{W}_{n j}^{3}, \bar{H}_{\mathrm{tan}}^{e}\left(\bar{J}_{n i}^{2 e}, 0\right)\right\rangle\right. \\
& \left.+\left\langle\bar{W}_{n j}^{3}, \bar{H}_{\mathrm{tan}}^{d}\left(\bar{J}_{n i}^{2 d}, 0\right)\right\rangle\right]+\eta_{e} \sum_{i=1}^{2\left(N_{d}-2\right)} K_{n i}\left[\left\langle\bar{W}_{n j}^{3}, \bar{H}_{\mathrm{tan}}^{e}\left(0, \bar{M}_{n i}\right)\right\rangle\right. \\
& \left.+\left\langle\bar{W}_{n j}^{3}, \bar{H}_{\mathrm{tan}}^{d}\left(0, \bar{M}_{n i}\right)\right\rangle\right]=\left\langle\bar{W}_{n j}^{3}, \bar{H}_{\mathrm{tan}}^{d}\left(\bar{J}^{i d}\right)\right\rangle \\
& \quad, \text { on } S_{d e}
\end{aligned}
$$


Eqs.(21) can be rearranged in submatrix form as follow:-

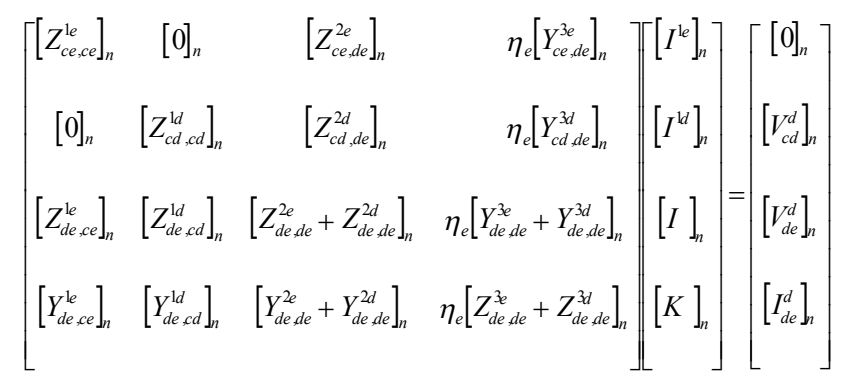

The superscripts 1,2 , and 3 on the impedance $Z$ and the admittance $Y$ submatrices represent the calculation of $Z$ and $Y$ due to $\bar{J}$ on the conductor, $\bar{J}$ on the dielectric, and $\bar{M}$ on the dielectric surfaces, respectively. The submatrices $Z$ and $Y$ with superscripts (e) and (d) denote the impedance and admittance matrices for the exterior or interior media, respectively. The first and second pairs of suffixes identify the field and source surfaces, respectively. The index $n$ implies the azimuthal mode number, and $V_{c d}^{d}, V_{d e}^{d}$, and $I_{d e}^{d}$ are the excitation submatrices caused by the electric and magnetic field sources on the surfaces $S_{c d}$ and $S_{d e}$ from the interior region. $I^{l e}$ and $I^{l d}$ are the unknown coefficients of the electric current density on the $S_{c e}$ and $S_{c d}$ surfaces, respectively. $I$ and $K$ are the unknown coefficients of the equivalent electric and magnetic current densities on the $S_{d e}$ surface.

Each submatrix of $Z$ or $Y$ in Eq. (22) consists of four submatrices. This equation can be written as follows:

$$
\left[\bar{T}_{n}\right]\left[\bar{I}_{n}\right]=\left[\bar{V}_{n}\right], n=0, \pm 1, \pm 2, \ldots \ldots
$$

where $\bar{T}_{n}$ is a square matrix representing the impedance and admittance submatrices, $\bar{I}_{n}$ is a column matrix for the unknown expansion coefficients of $\bar{J}$ and $\bar{M}$, and $\bar{V}_{n}$ is the excitation column matrix. Each mode has the matrix equations of the form in Eq. (22).

\section{2-4 Evaluation of the Matrix Element $\left[\bar{T}_{n}\right]$ :}

All elements of the T-matrix are given as the inner product of the weighting function with electric or magnetic fields caused by the electric and magnetic current densities.

\section{2-4-1 Evaluation of Z-Submatrices Elements}

In Eqs. (21), the mutual impedance between the source element $(i)$ and the observation element $(j)$ is taken from:-

(1) The electric field produced from the electric current density $\bar{J}$ :-

$$
\left(Z_{m n}^{\alpha \beta}\right)_{j i}^{J E}=\left\langle\bar{W}_{m j}^{\alpha}, \bar{E}^{a}\left(\bar{J}_{n i}^{\beta}, 0\right)\right\rangle
$$

where the electric field $\bar{E}^{a}\left(\bar{J}_{n i}^{\beta}, 0\right)$ can be expressed in terms of the magnetic vector and electric scalar potentials as follows :-

$$
\left(Z_{m n}^{\alpha \beta}\right)_{j i}^{J E}=\left\langle\bar{W}_{m j}^{\alpha},\left(-j \omega \bar{A}^{a}\left(\bar{J}_{n i}^{\beta}\right)-\nabla \phi^{a}\left(\bar{J}_{n i}^{\beta}\right)\right)\right\rangle
$$

(2) The magnetic field produced from the magnetic current density $\bar{M}$ :-

$$
\left(Z_{m n}^{\alpha \beta}\right)_{j i}^{M H}=\left\langle{\overline{W_{m j}}}_{j}, \bar{H}^{a}\left(0, \bar{M}_{n i}^{\beta}\right)\right\rangle
$$

where the magnetic field $\bar{H}^{a}\left(0, \bar{M}_{n i}^{\beta}\right)$ can be expressed in terms of the electric vector and magnetic scalar potentials as follows:-

$$
\left(Z_{m n}^{\alpha \beta}\right)_{j i}^{M H}=\left\langle\bar{W}_{m j}^{\alpha},\left(-j \omega \bar{F}^{a}\left(\bar{M}_{n i}^{\beta}\right)-\nabla \psi^{a}\left(\bar{M}_{n i}^{\beta}\right)\right)\right\rangle
$$

where $\alpha$ and $\beta$ are either the $t$ - or $\phi$-direction. The superscript JE denotes the impedance matrix found in the electric field and the electric current density, whereas the superscript $\mathrm{MH}$ refers to the impedance matrix found in the magnetic field and the magnetic current density. The relationship between these impedance matrices is proven in this paper as follows:-

$$
\left(Z_{n}^{\alpha \beta}\right)_{j i}^{M H}=\frac{1}{\eta_{a}^{2}}\left(Z_{n}^{\alpha \beta}\right)_{j i}^{J E}
$$

where $\eta_{a}=\sqrt{\mu_{a} / \varepsilon_{a}}$

The inner product of the electric field with the weighting function ${\overline{W_{m j}}}^{\alpha}$, [Eq. (20b)] provides the elements of the impedance matrix as follows:-

$$
\begin{aligned}
\left(Z_{n}^{t t}\right)_{j i}^{J E}= & -j K_{a} \eta_{a} \sum_{p=1}^{4} \sum_{q=1}^{4}\left[T _ { p } T _ { q } \left\{\sin v_{p} \sin v_{q} G_{2}\right.\right. \\
& \left.\left.+\cos v_{p} \cos v_{q} G_{1}\right\}-\frac{G_{1}}{K_{a}^{2}} T_{p}^{\prime} T_{q}^{\prime}\right] \\
\left(Z_{n}^{t \phi}\right)_{j i}^{J E}= & -K_{a} \eta_{a} \sum_{p=1}^{4} \sum_{q=1}^{4}\left[T_{p} T_{q} \sin v_{p} G_{3}+\frac{n}{K_{a}^{2}} \frac{T_{q}}{\rho_{q}} T_{p}^{\prime} G_{1}\right] \\
\left(Z_{n}^{\phi t}\right)_{j i}^{J E}= & K_{a} \eta_{a} \sum_{p=1}^{4} \sum_{q=1}^{4}\left[T_{p} T_{q} \sin v_{q} G_{3}+\frac{n}{K_{a}^{2}} T_{q}^{\prime} \frac{T_{p}}{\rho_{p}} G_{1}\right]
\end{aligned}
$$




$$
\left(Z_{n}^{\phi \phi}\right)_{j i}^{J E}=-j K_{a} \eta_{a} \sum_{p=1}^{4} \sum_{q=1}^{4} T_{p} T_{q}\left[G_{2}-\left(\frac{n}{K_{a}}\right)^{2} \frac{G_{1}}{\rho_{p} \rho_{q}}\right]
$$

where $G_{1}, G_{2}$ and $G_{3}$ are defined as:-

$$
\begin{aligned}
& G_{1}=\int_{0}^{\pi} \frac{e^{-j K_{a} R}}{R} \cos \left(n \phi^{\prime}\right) d \phi^{\prime} \\
& G_{2}=\int_{0}^{\pi} \frac{e^{-j K_{a} R}}{R} \cos \left(n \phi^{\prime}\right) \cos \phi^{\prime} d \phi^{\prime} \\
& G_{3}=\int_{0}^{\pi} \frac{e^{-j K_{a} R}}{R} \sin \left(n \phi^{\prime}\right) \sin \phi^{\prime} d \phi^{\prime} \\
& R=\sqrt{\left(\frac{\rho_{p}}{4}\right)^{2}+4 \rho_{p}^{2} \sin ^{2}\left(\frac{\phi^{\prime}}{2}\right)}
\end{aligned}
$$

The Gaussion quadrature technique is used to calculate these integrals. Eq. (28) represents the approximation for $R$ at $t=t^{\prime}$.

\section{2-4-2 Evaluation of the Y-Submatrices Elements}

In Eqs. (21), the mutual admittance between the source element $(i)$ and the observation element $(j)$ is taken from:-

(1) The magnetic field produced from the electric current density $\bar{J}$ :-

$$
\left(Y_{m n}^{\alpha \beta}\right)_{j i}^{J H}=\left\langle{\overline{W_{m j}}}_{m}^{\alpha}, \bar{H}^{a}\left(\bar{J}_{n i}^{\beta}, 0\right)\right\rangle
$$

The magnetic field can be expressed in terms of the magnetic vector potential, which yields the following:-

$$
\left(Y_{m n}^{\alpha \beta}\right)_{j i}^{J H}=\left\langle\bar{W}_{m j}^{\alpha},\left(\frac{1}{\mu_{a}} \nabla \times \bar{A}^{a}\left(\bar{J}_{n i}^{\beta}\right)\right)\right\rangle
$$

(2) The electric field produced from the magnetic current density $\bar{M}$ :-

$$
\left(Y_{m n}^{\alpha \beta}\right)_{j i}^{M E}=\left\langle\bar{W}_{m j}^{\alpha}, \bar{E}^{a}\left(0, \bar{M}_{n i}^{\beta}\right)\right\rangle
$$

The electric field can be expressed in terms of the electric vector potential. Thus, we have:-

$$
\left(Y_{m n}^{\alpha \beta}\right)_{j i}^{M E}=\left\langle\bar{W}_{m j}^{\alpha},\left(-\frac{1}{\varepsilon_{a}} \nabla \times \bar{F}^{a}\left(\bar{M}_{n i}^{\beta}\right)\right)\right\rangle
$$

where the superscript $\mathrm{JH}$ denotes the admittance matrix found in the magnetic field and the electric current density, whereas the superscripts ME refer to the admittance matrix found in the electric field and the magnetic cu- rrent density. The relationship between these admittance matrices is proven as follows:-

$$
\left(Y_{n}^{\alpha \beta}\right)_{j i}^{M E}=-\left(Y_{n}^{\alpha \beta}\right)_{j i}^{J H}
$$

The dot product of the magnetic field with the weighting function ${\overline{W_{m j}}}^{\alpha}$ gives the elements of the admittance matrix as follows:-

$$
\begin{aligned}
\left(Y_{n}^{t t}\right)_{j i}^{J H}= & j \sum_{p=1}^{4} \sum_{q=1}^{4} T_{p} T_{q} G_{6}\left[\rho_{q} \cos v_{q} \sin v_{p}\right. \\
& \left.+\sin v_{q}\left\{\sin v_{p}\left(z_{p}-z_{q}\right)-\rho_{p} \cos v_{p}\right\}\right] \\
\left(Y_{n}^{t \phi}\right)_{j i}^{J H}= & \sum_{p=1}^{4} \sum_{q=1}^{4} T_{p} T_{q}\left[\rho_{q} \cos v_{p} G_{4}\right. \\
& \left.+\left\{\sin v_{p}\left(z_{p}-z_{q}\right)-\rho_{p} \cos v_{p}\right\} G_{5}\right] \\
\left(Y_{n}^{\phi t}\right)_{j i}^{J H}= & \sum_{p=1}^{4} \sum_{q=1}^{4} T_{p} T_{q}\left[\rho_{p} \cos v_{q} G_{4}\right. \\
& \left.-\left\{\sin v_{q}\left(z_{p}-z_{q}\right)+\rho_{q} \cos v_{q}\right\} G_{5}\right] \\
\left(Y_{n}^{\phi \phi}\right)_{j i}^{J H} & =j \sum_{p=1}^{4} \sum_{q=1}^{4} T_{p} T_{q}\left(z_{p}-z_{q}\right) G_{6}
\end{aligned}
$$

where,

$$
\begin{aligned}
G_{4} & =\int_{0}^{\pi} \cos \left(n \phi^{\prime}\right) \frac{\left(1+j K_{a} R\right)}{R^{3}} e^{-j K_{a} R} d \phi^{\prime} \\
G_{5} & =\int_{0}^{\pi} \cos \left(n \phi^{\prime}\right) \cos \phi^{\prime} \frac{\left(1+j K_{a} R\right)}{R^{3}} e^{-j K_{a} R} d \phi^{\prime} \\
G_{6} & =\int_{0}^{\pi} \sin \left(n \phi^{\prime}\right) \sin \phi^{\prime} \frac{\left(1+j K_{a} R\right)}{R^{3}} e^{-j K_{a} R} d \phi^{\prime}
\end{aligned}
$$

Again, the Gaussion quadrature techniques are used to evaluate the integrals of Eqs. (33).

Here, $\rho_{p}, \rho_{q}, v_{p}$, and $v_{q}$ are the $\rho$ and $v$ evaluated at $t_{p}$ and $t_{q}$, respectively, where $t_{p}$ and $t_{q}$ are given by:-

$$
t_{p}=j+\frac{p-2.5}{2}, \quad t_{q}=i+\frac{p-2.5}{2}
$$

\section{2-4-3 Evaluation of the Excitation Submatrices Ele- ments}

The excitation fields $\bar{E}_{\text {tan }}^{d}\left(\bar{J}^{i d}\right)$ and $\bar{H}_{\text {tan }}^{d}\left(\bar{J}^{i d}\right)$ are defined as the fields of finite sources within the dielectric region; they radiate in an unbounded region. The elements of the excitation matrix are the fields caused by the current elements located in the dielectric substrate, 
which are $\left(V_{c d}^{d}\right)_{n j},\left(V_{d e}^{d}\right)_{n j}$, and $\left(I_{d e}^{d}\right)_{n j}$, calculated at the generating surface of the MSA (i.e., $S_{c d}+S_{d e}$ ). The elements of the excitation matrix are defined as the inner products of $\bar{E}_{\text {tan }}^{d}\left(\bar{J}^{i d}\right)$ or $\bar{H}_{\text {tan }}^{d}\left(\bar{J}^{i d}\right)$ with testing functions $\bar{W}_{n j}(\bar{r})$. These elements are shown in Eqs. (21) as follows:-

$$
\begin{aligned}
& \left(V_{n}^{d}\right)_{j}^{\alpha}=\int_{s} \bar{W}_{n j}^{\alpha} \cdot \bar{E}_{\text {tan }}^{i n c}\left(\bar{J}^{i d}\right) d s \\
& \left(I_{n}^{d}\right)_{j}^{\alpha}=\int_{s} \bar{W}_{n j}^{\alpha} \cdot \bar{H}_{\text {tan }}^{i n c}\left(\bar{J}^{i d}\right) d s
\end{aligned}
$$

where $\alpha$ is the $t$ or $\phi$-component.

The electric and magnetic radiation fields caused by an electric current element are given by:-

$$
\begin{aligned}
& \bar{E}^{i n c}\left(\bar{I}^{i d}\right)=-j \omega \bar{A}^{d}\left(\bar{I}^{i d}\right)-\nabla \Phi^{d}\left(\bar{I}^{i d}\right) \\
& \bar{H}^{i n c}\left(\bar{I}^{i d}\right)=\frac{1}{\mu_{d}} \nabla \times \bar{A}^{d}\left(\bar{I}^{i d}\right)
\end{aligned}
$$

where $\bar{A}^{d}\left(\bar{I}^{i d}\right)$ is the magnetic vector potential, and $\Phi^{d}\left(\bar{I}^{i d}\right)$ is the electric scalar potential given by :-

$$
\begin{aligned}
& \bar{A}^{d}\left(\bar{I}^{i d}\right)=-\frac{j K_{d} \mu_{d}}{4 \pi} \bar{I}^{i d} \ell h_{0}^{(2)}\left(K_{d} \mid \bar{r}-\bar{r}^{\prime}\right) \\
& \Phi^{d}\left(\bar{I}^{i d}\right)=\frac{\eta_{d}}{4 \pi} \bar{I}^{i d} \ell \cdot \nabla h_{0}^{(2)}\left(K_{d} \mid \bar{r}-\bar{r}^{\prime}\right)
\end{aligned}
$$

where $\bar{I}^{i d} \ell$ is the dipole moment in the $\hat{u}_{z}$ - direction, and $h_{0}^{(2)}\left(K_{d} \mid \bar{r}-\bar{r}^{\prime}\right)$ is the spherical Hankel function of the second kind zero order.

The solution of Eqs. (35) provides the following elements of the excitation matrix:-

$$
\begin{aligned}
& \left(V_{n}^{d}\right)_{j}^{t}=-j \frac{K_{d} \eta_{d}}{2 \pi} I^{i d} \ell \sum_{p=1}^{4}\left[\cos v_{p} T_{p} G_{V 1}+\frac{z_{p}}{K_{d}^{2}} T_{p}^{\prime} G_{V 2}\right] \\
& \left(V_{n}^{d}\right)_{j}^{\phi}=-\frac{n}{2 \pi} \frac{\eta_{d}}{K_{d}} I^{i d} \ell \sum_{p=1}^{4}\left[\frac{T_{p}}{\rho_{p}} z_{p} G_{V 2}\right] \\
& \left(I_{n}^{d}\right)_{j}^{t}=-j \frac{\rho_{f}}{2 \pi} I^{i d} \ell \sum_{p=1}^{4}\left[\sin v_{p} T_{p} G_{V 3}\right] \\
& \left(I_{n}^{d}\right)_{j}^{\phi}=\frac{I^{i d} \ell}{2 \pi} \sum_{p=1}^{4} T_{p}\left[\rho_{p} G_{V 2}-\rho_{f} G_{V 4}\right]
\end{aligned}
$$

where,

$$
\begin{aligned}
G_{V 1} & =\int_{0}^{\pi} \cos (n \phi) \frac{e^{-j K_{d} R_{f}}}{R_{f}} d \phi \\
G_{V 2} & =\int_{0}^{\pi} \cos (n \phi) G_{f}\left(\bar{r}, \bar{r}^{\prime}\right) d \phi \\
G_{V 3} & =\int_{0}^{\pi} \sin (n \phi) \sin \left(\phi_{f}-\phi\right) G_{f}\left(\bar{r}, \bar{r}^{\prime}\right) d \phi \\
G_{V 4} & =\int_{0}^{\pi} \cos (n \phi) \cos \left(\phi_{f}-\phi\right) G_{f}\left(\bar{r}, \bar{r}^{\prime}\right) d \phi
\end{aligned}
$$

and

$$
\begin{aligned}
& G_{f}\left(\bar{r}, \bar{r}^{\prime}\right)=\left(\frac{1+j K_{d} R_{f}}{R_{f}^{3}}\right) e^{-j K_{d} R_{f}} \\
& R_{f}=\left[z_{p}^{2}+\rho_{p}^{2}+\rho_{f}^{2}-2 \rho_{p} \rho_{f} \cos \left(\phi_{f}-\phi\right)\right]^{1 / 2}
\end{aligned}
$$

where $\rho_{f}$ and $\phi_{f}$ are the coordinates of the feed point. The parameters $\rho_{p}, z_{p}$, and $v_{p}$ are the coordinates of the field point on the surfaces $S_{c d}$ or $S_{d e}$. They are assumed to be constant in each pulse and equal to their values at the midpoint of the pulse. $T_{p}$ and $T_{p}^{\prime}$ are the weighting factors of the impulse functions approximating the triangular functions and their derivatives.

\section{2-5 Evaluation of the Radiation Fields}

The radiation fields of the CMSA are calculated from a knowledge of the equivalent electric and magnetic surface current densities flowing over the outer surfaces of the ground plane, dielectric and patch. Once, these surface current densities $\bar{J}$ and $\bar{M}$ are determined, then the far-field components $E_{\theta}$ and $E_{\phi}$ can be obtained from :-

$$
\begin{aligned}
& E_{\theta}\left(\theta_{o}, \phi_{o}\right)=-\frac{j w \mu_{e}}{4 \pi r_{o}} e^{-j K_{e} r_{o}} \sum_{n=0}^{\infty}\left(\sum_{i=1}^{N-6}\left[\left(R_{n}^{t \theta}\right)_{i} I_{n i}^{t}+\left(R_{n}^{\phi \theta}\right)_{i} I_{n i}^{\phi}\right]\right. \\
& \left.+\eta_{r} \sum_{i=1}^{N_{d}-}\left[\left(R_{n}^{t \phi}\right)_{i} K_{n i}^{t}+\left(R_{n}^{\phi \phi}\right)_{i} K_{n i}^{\phi}\right]\right] \\
& E_{\phi}\left(\theta_{o}, \phi_{o}\right)=-\frac{j w \mu_{e}}{4 \pi r_{o}} e^{-j K_{e} r_{o}} \sum_{n=0}^{\infty}\left(\sum_{i=1}^{N-6}\left[\left(R_{n}^{t \phi}\right)_{i} I_{n i}^{t}+\left(R_{n}^{\phi \phi}\right)_{i} I_{n i}^{\phi}\right]\right. \\
& \left.-\eta_{r} \sum_{i=1}^{N_{d}-2}\left[\left(R_{n}^{t \theta}\right)_{i} K_{n i}^{t}+\left(R_{n}^{\phi \theta}\right)_{i} K_{n i}^{\phi}\right]\right)
\end{aligned}
$$

Where,

$$
\begin{aligned}
\left(R_{n}^{t \theta}\right)_{i}= & \sum_{q=1}^{4} C\left[j \sin v_{q} \cos \theta_{o}\left(J_{n+1}(u)-J_{n-1}(u)\right)\right. \\
& \left.-2 \cos v_{q} \sin \theta_{o} J_{n}(u)\right] \\
\left(R_{n}^{\phi \theta}\right)_{i}= & -\sum_{q=1}^{4} C \cos \theta_{o}\left[J_{n+1}(u)+J_{n-1}(u)\right] \\
\left(R_{n}^{t \phi}\right)_{i}= & \sum_{q=1}^{4} C \sin v_{q}\left[J_{n+1}(u)+J_{n-1}(u)\right] \\
\left(R_{n}^{\phi \phi}\right)_{i}= & j \sum_{q=1}^{4} C\left[J_{n+1}(u)-J_{n-1}(u)\right] \\
C= & j^{n} \pi e^{j n \phi_{o}} e^{j K_{e} z_{q} \cos \theta_{o}} T_{q}\left(t^{\prime}\right) \\
u= & K_{e} \rho_{q} \sin \theta_{o}
\end{aligned}
$$

and $J_{n}(u), J_{n-1}(u)$ and $J_{n+1}(u)$ are the Bessel functions of the first kind and order $(n),(n-1)$, and $(n+1)$, respec- 


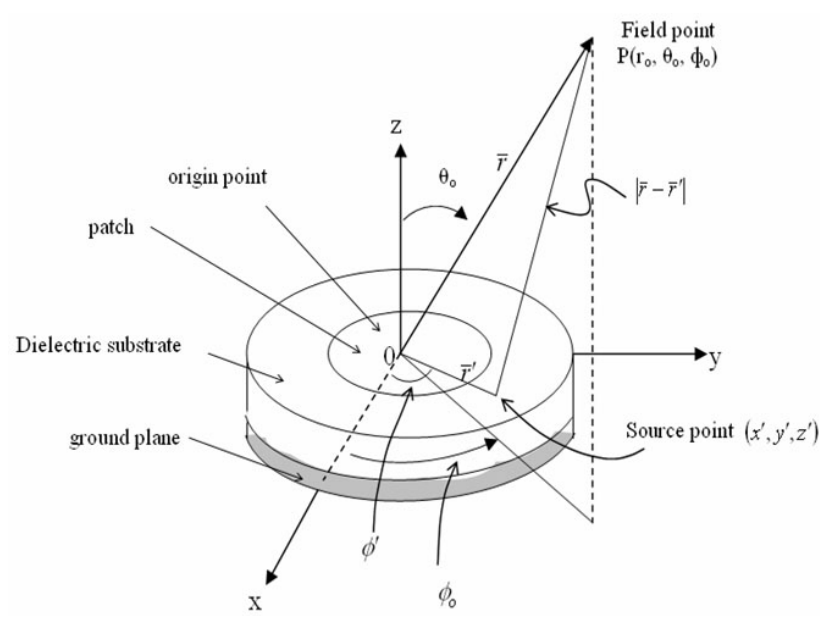

Fig. 5. Relationship between source and field points.

tively.

Where $r_{o}, \theta_{o}$, and $\phi_{o}$ are the units vectors of the coordinates shown in Fig. 5, and the integrals are carried out over the total outer surface of the body.

\section{Results and Discussion}

The surface currents are determined and plotted for both the dominant $\mathrm{TM}_{11^{-}}$and $\mathrm{TM}_{21^{-}}$modes. Fig. (6) shows the computed electric and magnetic surface currents for the $\mathrm{TM}_{11}$-mode at the outer boundary of the microstrip surface. The horizontal axis shows the length of the contour $(L)$ along the generating curve. Due to the structure symmetry, only half of the geometry is considered. In this figure, the external surface currents of the three regions are plotted with respect to their locations on the surface, where these regions correspond to the ground plane, the dielectric substrate, and the patch surface, respectively. An examination of this figure reveals that the electric current is the strongest on the patch surface and has a negligible value on the ground plane. The $t$-component of the electric current value is small on the dielectric, whereas the $\phi$-component of this current is considerably stronger than the $t$-component and is maximum near the patch edge. The magnetic current $M^{\phi}$ is considerably stronger than $M^{t}$; it is maximum near the patch edge and negligible in terms of the dielectric thickness. Therefore, the contributions to the antenna radiation mainly come from the $\phi$-components of the electric and magnetic currents on the dielectric surface near the patch edge and the electric current on the upper patch surface.

Fig. (7) shows that the $t$-component of the electric surface current for the $\mathrm{TM}_{21}$-mode has a small value on the outside boundary except near the center of the patch, whereas the $\phi$-component of this current has the high-

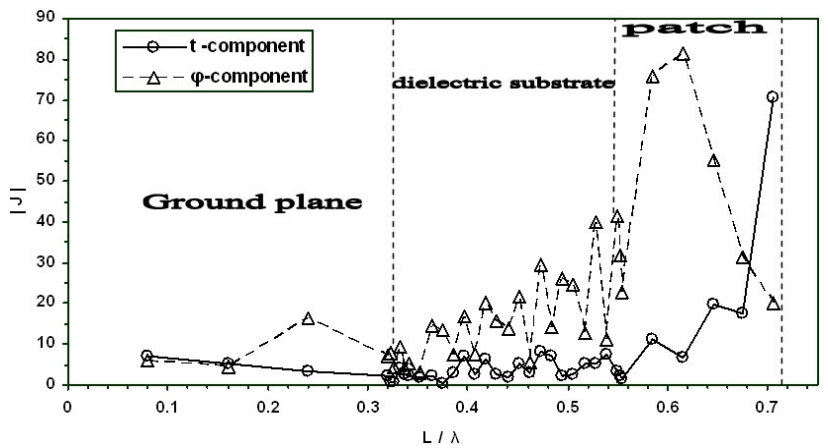

(a)

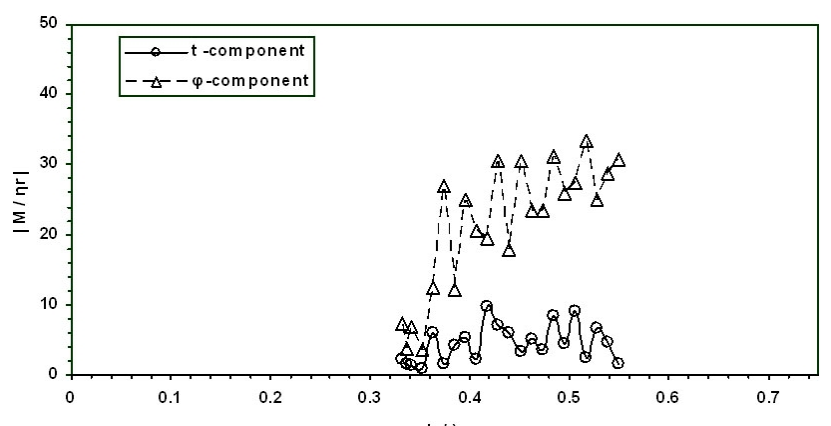

(b)

Fig. 6. Electric and magnetic surfaces current densities for the $\mathrm{TM}_{11}$-mode on the outside boundary of a CMSA $\left(g=0.4 \lambda, h=0.02 \lambda, f_{r}=3.214 \mathrm{GHz}, \varepsilon_{r}=2.32, g_{t}\right.$ $=0.01 \lambda, a_{t}=0.00775 \lambda, \rho_{f}=0.05 \lambda, \phi_{f}=25^{\circ}$ ).

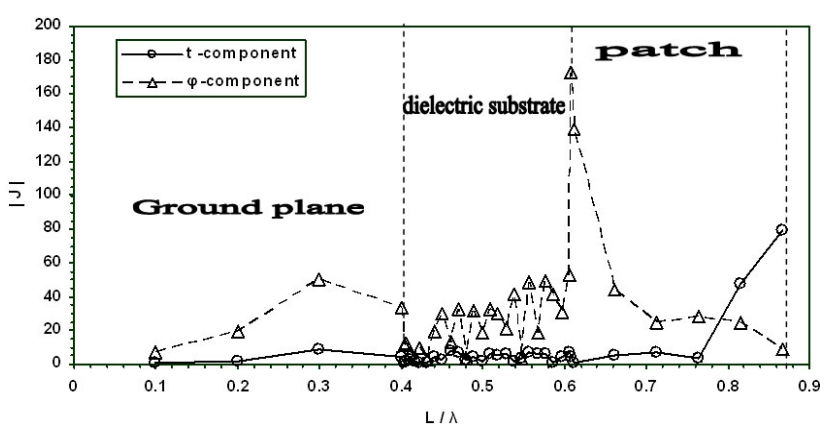

(a)

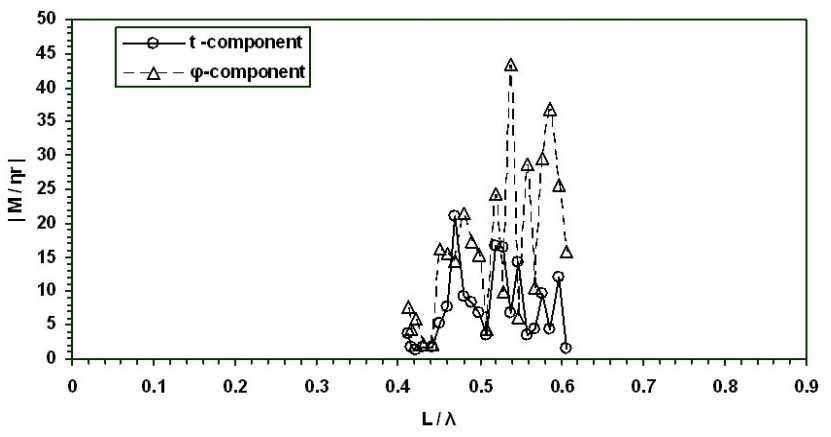

(b)

Fig. 7. Electric and magnetic surfaces current densities for the $\mathrm{TM}_{21}$-mode on the outside boundary of a CMSA $\left(g=0.5 \lambda, h=0.02 \lambda, f_{r}=3.214 \mathrm{GHz}, \varepsilon_{r}=2.32, g_{t}\right.$ $=0.01 \lambda, a_{t}=0.00775 \lambda, \rho_{f}=0.23 \lambda, \phi_{f}=0^{\circ}$ ). 

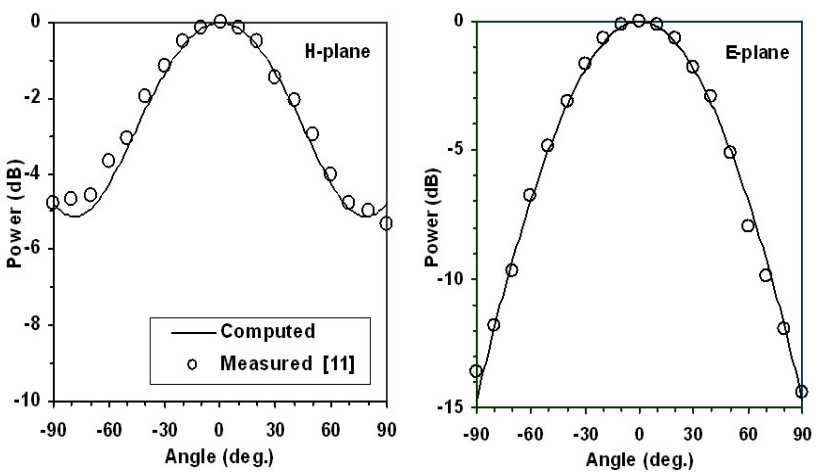

(a) $\left(g=0.31 \lambda, h=0.00397 \lambda, \varepsilon_{r}=794 \mathrm{MHz}, \mathcal{E}_{r}=2.32\right.$, $g_{t}=0.01 \lambda, a_{t}=0.0077 \lambda, \rho_{f}=0.05 \lambda, \phi_{f}=0^{\circ}$ )
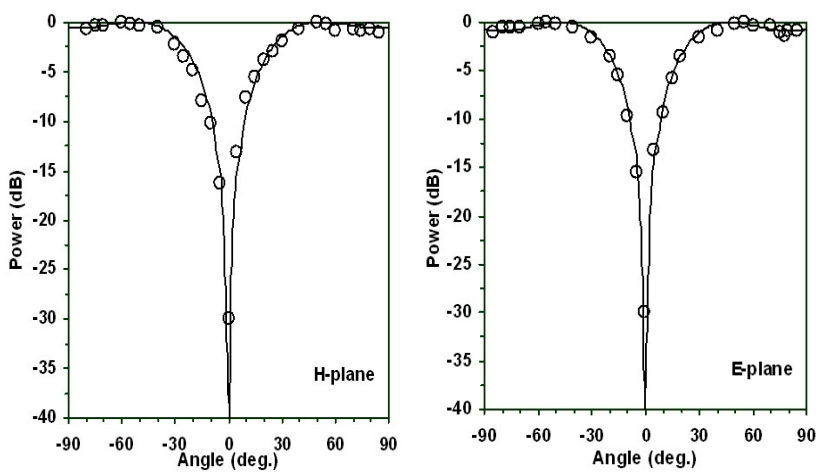

(b) $\left(g=0.435 \lambda, h=0.00662 \lambda, f_{r}=1,324 \mathrm{MHz}, \varepsilon_{r}=2.32\right.$, $g_{t}=0.01 \lambda, a_{t}=0.01 \lambda, \rho_{f}=0.22 \lambda, \phi_{f}=0^{\circ}$ )

Fig. 8. Radiation patterns of a CMSA excited by, (a) $\mathrm{TM}_{11^{-}}$ mode (b) $\mathrm{TM}_{21}$-mode.

est value on these boundaries. The magnetic current components $\left(M^{t}, M^{\phi}\right)$ and the main radiation zones are the same as those in the $\mathrm{TM}_{11}$-mode case.

In any program, to develop techniques for solving radiating problems, it is necessary to verify the techniques and examine the accuracy of the results by comparing it with well-known results.

For the purpose of validity and convergence, Fig. 8 shows the radiation patterns in the principle plane for a CMSA compared with the experimental results of Lo Y. T. [11].

It can be seen from this figure that the theoretical results agrees well with the measured results.

\section{Conclusions}

The analytical treatment of this work enables us to include the effects of the adjacent modes on the dominant mode in calculating the surface current of the CMSA. The computed electric current density $J$ at the outside boundary of the antennas under study is the strongest on the patch surface and has a negligible value on the ground plane and dielectric surfaces. Note also that the radial component $J^{t}$ of this density increases rapidly as it approaches the center of the patch, whereas the angular component $J^{\phi}$ of this current is considerably larger than $J^{t}$ and is maximum near the patch edge. The magnetic component $M^{\phi}$ is considerably stronger than $M^{t}$ and is maximum near the patch edge; these components are negligible in terms of the dielectric thickness.

\section{References}

[1] I. J. Bahl, P. Bahartia, Microstrip Antennas, Artech House Inc., 1980.

[2] D. D. Sandu, O. G. Avadanei, A. Ioachim, and D. Ionesi, "Contribution to the cavity model for analysis of microstrip patch antennas," Journal of Optoelectronics and Advanced Materials, vol. 8, no. 1, pp. 339-344, 2006.

[3] K. Alexander, "Aperture-coupled microstrip patch antenna array," Diplomarbeit, Friedhofallee 4a/11, A2232 Deutsch-Wagram, 1996.

[4] R. F. Harrington, Field Computation by Moment Methods, Macmillan, New York, 1968.

[5] R. Garg, P. Bhartia, I. Bahl, and A. Ittipiboon, Microstrip Antenna Design Handbook, Artech House Inc., 2001.

[6] A. A. Kishk, L. Shafai, "Numerical solution of scattering from coated bodies of revolution using different integral equation formulations," IEE Proc., vol. 133, Pt. H. no. 3, pp. 227-232, 1986.

[7] J. R. James, P. S. Hall, Handbook of Microstrip Antennas, Peter Peregrines, London, U. K., 1989.

[8] L. N. Medgyesi-Mitschang, J. H. Mullen,, "Radiation and scattering from asymmetrically excited bodies of revolution," IEEE Trans. Anten. and Propag., pp. 90-93, 1976.

[9] K. A. Iskander, L. Shafai, A. Frandsen and J. E. Hansen, "Application of impedance boundary conditions to numerical solution of corrugated circular horns," IEEE Trans. Anten. and Propag., vol. AP-30, no. 3, pp. 366-372, 1982.

[10] P. L. Huddleston, L. N. Medgyesi-Mitschang, and J. M. Putnam, "Combined field integral equation formulation for scattering by dielectrically coated conducting bodies," IEEE Trans. Anten. and Propag., vol. Ap-34, no. 4, pp. 510-520, 1986.

[11] Y. T. Lo, D. Solomon, and W. F. Richards, "Theory and experiment on microstrip antennas," IEEE Trans. Anten. and Propag., vol. AP-27, no. 2, pp. 137-146, 1979. 


\section{Wa'il A. Godaymi}

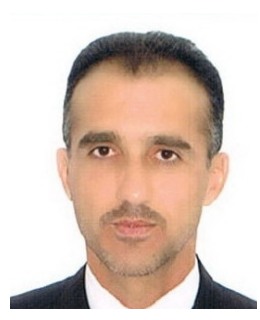

was born in Iraq on September 8, 1971. He received the B.Sc. degree in physics, M. Sc. degree in microwave antennas and $\mathrm{Ph}$. D. degree in electromagnetic computation from the University of Basra, Basrah, Iraq, in 1995, 1999 and 2008, respectively. $\mathrm{He}$ is now Assistant Professor at Basra University of Iraq. His research interest include microstrip antennas, circularly polarized antennas, antennas array, radar cross section, and numerical methods in electromagnetic.

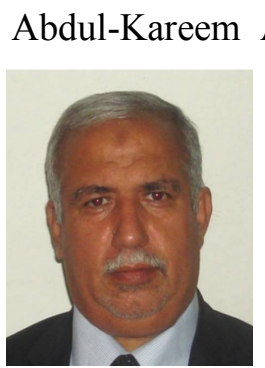

Abd Ali Mohammed

was born in AL Nassiria, Iraq, in 1958. $\mathrm{He}$ received his $\mathrm{BSc}$ in electrical engineering (1980) from Sulaimania University, Sulaimania, Iraq, postgraduate diploma in communications (1982) and MSc in communication (1984) from the University of Technology, Baghdad, Iraq. From 1984 to 1988 he was working with the Electromagnetic Wave Propagation Department, Space and Astronomy Research Center, Scientific Research Council, Baghdad, Iraq. From 1988 to 1993 he was working with the Space Technology Department, Space Research Center, Baghdad, Iraq. On, 1994, he joined the Physics department, college of science, Saddam University, Baghdad, Iraq where he obtained his Ph.D. (1997) in electromagnetic, microstrip microwave antennas. From 1997 to 2003 he was working with the Al-Battany Space Directorate as a researcher and head of group in the field of microwave system. Since 2004 he is the head of Space and Atmosphere Research Center in Iraqi ministry of science and technology. Now he leads group of atmosphere remote sensing for dust storm monitoring and detection by using different space tools. Since January 2011 he joined a post doctorate in Systems Engineering Department, University Arkansas at Little Rock in the field of dust storm monitoring.

\section{Zeki A. Ahmed}

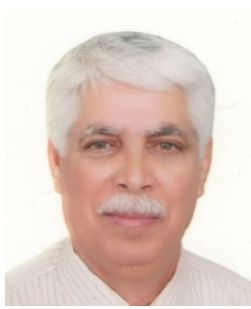

was born in Basra-Iraq on December 1954. He received the B.Ss degree in physics, M.Sc in 1983 and Ph.D. in 1996 in Antennas from University of Basra. He has worked from 1984 till 1992 as: assistant lecturer, lecturer and assistant professor in Department of Physics, College of Science, University of Basra, in Iraq. $\mathrm{He}$ is now a professor in microwave antennas, and works in the same Department. His research interest are microstrip antennas and the radiation and scattering problems of electromagnetic. 